\title{
Instantaneous heat transfers at the impact of a droplet onto a hot surfaces in the film boiling regime
}

\author{
William Chaze ${ }^{1,2}$, Guillaume Castanet ${ }^{*, 1,2}$, Ophélie Caballina ${ }^{1,2}$, Denis Maillet ${ }^{1,2}$, \\ Jean-François Pierson ${ }^{3}$, Fabrice Lemoine ${ }^{1,2}$ \\ ${ }^{1}$ LEMTA, UMR 7563, Université de Lorraine, Vandœuvre-Lès-Nancy, France \\ ${ }^{2}$ LEMTA, UMR 7563, CNRS, Vandœuvre-Lès-Nancy, France \\ ${ }^{3}$ Institut Jean Lamour, UMR CNRS 7198, Université de Lorraine, Nancy, France \\ ${ }^{\star}$ Corresponding author: guillaume.castanet@univ-lorraine.fr
}

\begin{abstract}
Heat and mass transfers at the impact of a droplet onto a hot solid surface are investigated experimentally. Millimetersized water droplets impinges onto a perfectly flat sapphire surface heated at $600^{\circ} \mathrm{C}$. The temperature of the liquid inside the droplet is measured using the two-color laser-induced fluorescence (2cLIF) technique. Water is seeded with a temperature-sensitive fluorescent dye, while a nanosecond pulsed laser is used for the excitation of the fluorescence. The ratio of fluorescence signal detected in two appropriate spectral bands allows to determine the liquid temperature. One advantage of this non-intrusive optical technique is that it eliminates adverse effects associated with signal variations caused by droplet shape during its impact. In parallel, the temperature of the solid surface is characterized using infrared thermography. The latter measurements are made possible by the deposition of a nanosize coating of titanium aluminium nitride (TiAIN) on the upper surface of the sapphire window. Thanks to the high frame rate of the IR camera, the time evolution of the heat flux distribution at the solid surface can be reconstructed. A comparison of IR and 2cLIF techniques enable to correlate the heating of the liquid with the cooling of the wall. This reveals that most of the heat removed from the solid surface is devoted to the heating of the liquid, the energy used for liquid vaporization being significantly lower.
\end{abstract}

\section{Introduction}

Many industrial applications require a rapid cooling of surfaces from high temperatures. Among the cooling technologies, spray cooling is certainly one of the most attractive for the thermal management of high heat flux systems. Compared to jet impingement, it has the capability of cooling a relatively wider surface area with a single nozzle. It also has an unrivalled cooling efficiency, meaning that significant quantities of coolant liquid can be saved to remove the same amount of heat. These features explain why it is widely employed in many industrial applications, especially in metal production and processing industry. However, while it is applied for decades, its integration remains a complex and cumbersome process because of still incomplete knowledge of the fluid flow and heat transfer characteristics. In particular, scientific investigations focused on individual droplets are still required to understand the underlying physics behind the interactions between droplets and a hot solid surface. When a drop impacts a hot wall, different behaviours are observed. The drop can spread over the solid surface and remain attached to it due to wettability forces. It can splash and creates several smaller secondary droplets or simply rebounds. Extensive experimental investigations were carried out in the past to characterize the parameters influencing the drop behavior at the impact. Among them, some can be related to the dynamic of the impacting droplets (velocity, diameter, etc.), the physical properties of the liquid (viscosity, surface tension, etc.), and the solid surface (temperature, roughness, thermal effusivity, etc.) [1]. Descriptions of an impact are usually made on the basis of correlations with dimensionless numbers characterizing the relative magnitude of the forces acting on the impinging droplet, i.e. Reynolds, Weber and Ohnesorge numbers.

Regarding heat transfers, almost all the studies were focused around the heat removal from the solid surface, whose temperature was monitored using either thermocouples embedded in the wall thickness [2] and more recently by IR thermography $[3,4]$. Coupled with an inverse model, these measurements made quantification of the heat removed from the wall and the cooling efficiency [4] possible .Recently, Jung et al. [3] used IR thermography to characterize the temporal evolution of the heat flux at the wall during the impact of millimeter-size water droplets. A sapphire window, transparent in the IR up to $5 \mu \mathrm{m}$, is coated with a nanometer layer of platinum on the impact surface. Direct observation of the platinimum deposit through the sapphire allows to reconstruct the heat flux evolution with almost no limitation in temporal resolution other than the frame rate of the IR camera. Even though platinum is very reflective and therefore not very emissive $(\varepsilon \approx 0.04)$, accurate measurements of the surface temperature could be achieved.

The contributions of liquid evaporation and liquid heating to the overall heat exchange between the droplet and the wall are not obvious to evaluate if measurements are restricted to the wall heat flux. The droplet heating can be characterized using the two-color laser-induced fluorescence thermometry (2cLIF) which is one of the few proven techniques available to measure the temperature of droplets [5]. The liquid (in this case water) is seeded by a temperature-dependent fluorescent dye. The capabilities of the technique for studying the drop impact was initially demonstrated on mono-sized droplet streams impinging obliquely a heated wall [6]. Dunand et al. [7] made use of a laser sheet and two cameras in order to visualize the temperature of the impacting droplets. However, using a $\mathrm{CW}$ laser, measurements suffer from serious limitations. Light intensity within the laser sheet was not sufficient to 
shorten the exposure time of the cameras enough to obtain instantaneous images of the impinging droplets. More recently, several improvements to the 2cLIF technique were made by [8] to achieve single shot measurements of the temperature using nanosecond pulse laser.

The present study relies on these previous works for the development of optical diagnostics adapted to the characterization of heat and mass transfers at the drop impact on heated solid surfaces. The focus is put on the film boiling regime, in which a thin vapor film at the interface between the droplet and the solid surface prevents the deposition of liquid on the wall surface. Ultimately, the experimental results will help understanding the coupling between the droplet deformation at the impact and the heat and mass transfers. They will also be useful benchmarks to validate detailed numerical simulations which are taking into account phase change and heat transfers.

\section{Experimental set-up}

The experimental setup is presented in Figure 1. Millimeter-sized drops are generated in a very reproducible manner by a syringe. When the drop pending to the end of the needle exceeds a certain size, it detaches itself. A $400 \mu \mathrm{m}$ base diameter needle is used to produce water drops with a diameter of $2.5 \mathrm{~mm}$. The frequency of the droplet detachment is controlled by means of a syringe drivers which allows delivering a constant liquid flow rate. The free-falling droplets impinge a sapphire window (1" in diameter and $5 \mathrm{~mm}$ thick) placed on a steel holder which is heated at $600^{\circ} \mathrm{C}$ using cartridges heaters $(4 \times 250 \mathrm{~W})$. Owing to the large thermal conductivity of sapphire (about $40 \mathrm{~W} / \mathrm{m} / \mathrm{K}$ ), the temperature of the solid surface is uniform and and nearly equal to that of the steel holder. A thermal shield is necessary to prevent heating of the liquid inside the needle caused by the hot air plume rising from the sapphire window. The tip of the needle is placed into a cavity of a few millimeters arranged inside a metallic plate which is cooled down by a water circulation. Moreover, the fluorescent solution passes into a heat exchanger before entering the needle. A thermocouple, placed at

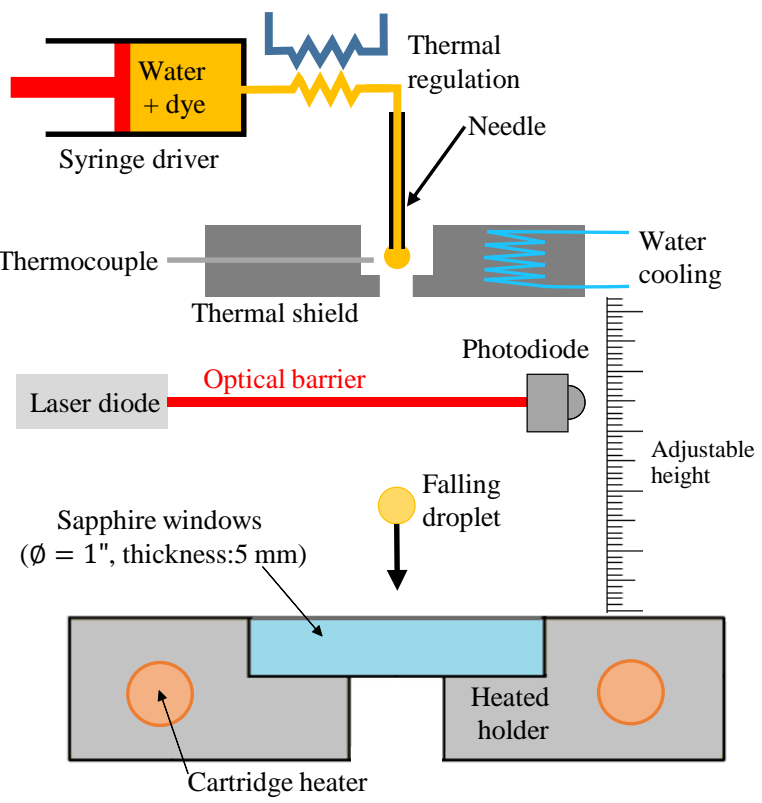

Figure 1. The experimental setup a short distance from the droplet in the cavity, is used to control the ambient temperature. This provides a good approximation for the temperature of the pendant droplet, because the same setpoint is imposed in the thermal regulations with the heat exchanger and the thermal shield. The needle is moved up and down to modify the impact velocity. Finally, an aperture managed in the steel holder allows using a sapphire window for a backside illumination and/or visualization of the impinging droplet.

\section{Droplet temperature measurements}

A pulsed Nd: YAG laser at $532 \mathrm{~nm}$ (Quantel Brillant B, pulse energy $E=450 \mathrm{~mJ}$, pulse duration $d t=5 \mathrm{~ns}$ ) is utilized for the excitation of the fluorescence. The laser beam is much larger than the droplets, which yields an illumination of the whole liquid volume during the droplet deformation. The repetition rate of the pulsed laser $(10 \mathrm{~Hz})$ is by far too low to resolve temporally the impact process. However, a time reconstruction is possible owing to the fact that droplets can be produced repeatedly with exactly the same size. An optical barrier consisting of a laser diode and a photodiode, placed a few millimeters above the sapphire surface, makes the detection of the fall of a droplet possible. The time delay between the detection by the barrier and the triggering of the laser pulse is varied by a small increment in order to explore, droplet after droplet, the whole period of an impact. As displayed in Figure 2, droplets are observed by means of two CCD cameras (Allied Vision Tech Prosilica GT3300 B/C GigE Camera $3296 \times 2472,12$ bits, $5.5 \mu \mathrm{m}$ ) each one equipped with an interference filter for the detection of the fluorescence in a specific spectral band. The optical system also includes an objective lens (SIGMA APO MACRO $150 \mathrm{~mm}$ F2.8 EX DG OS HSM and its teleconverter $\times 2$ ) and a beamsplitter mounted in front of the cameras. A high pass filter $(\lambda>542 \mathrm{~nm})$ is added between the objective lens and the beamsplitter to eliminate more efficiently the laser light. Examples of temperature measurements based on 2cLIF can be found in the literature. Application are related to single-phase liquid flows [8], monosized droplet streams [7] and sprays [5]. In most of these applications, a CW laser was used to induce fluorescence. However, for the purpose of instantaneous imaging, it is very advantageous using pulsed laser sources, which allow delivering a considerable amount of energy over a very short period of time (typically a few ns). According to Chaze et al.[8], the fluorescence $F_{\lambda}$ signal emitted at a wavelength $\lambda$ in an elementary volume $V$ of liquid can be expressed by:

$$
F_{\lambda}=\eta \frac{\Omega}{4 \pi} \varepsilon_{0} \phi_{\lambda} \frac{I_{0}}{\left(1+I_{0} / I_{\text {sat }}\right)} C V .
$$

In this expression, $I_{0}$ corresponds to the laser intensity, $C$ is the molar concentration of the dye molecules. $\eta$ is the transmission efficiency of the fluorescence light to the detector. $\Omega$ denotes the solid angle of the collection. $\varepsilon_{0}$ is the molar extinction coefficient of the fluorescent molecules at the excitation wavelength of the laser beam. $\phi_{\lambda}$ is 


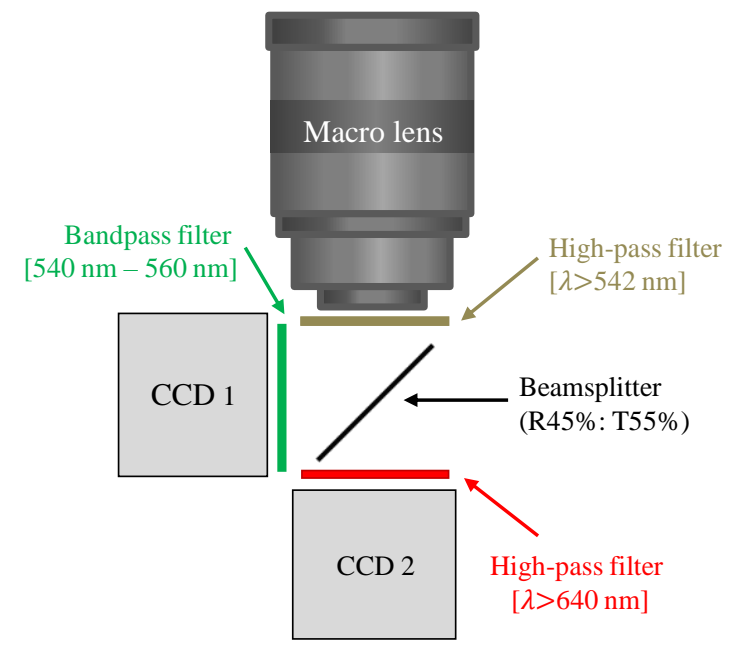

Figure 2. Arrangement of the optical detection system of the 2cLIF technique

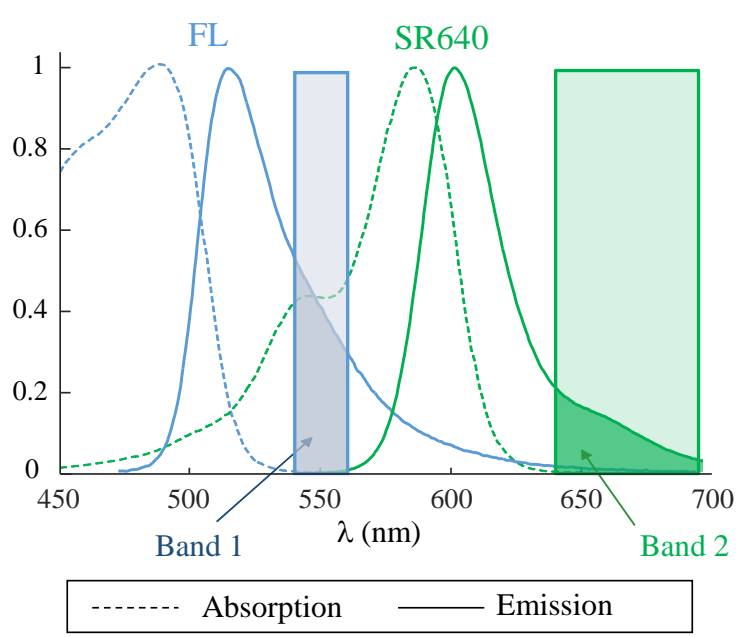

Figure 3. The two detection bands selected for the application of the 2cLIF technique using the couple of dyes

to the fluorescence quantum yield. $I_{\text {sat }}$ is the saturation intensity of the fluorescent dye (typically a few $\mathrm{MW} / \mathrm{cm}^{2}$ [8]). In Eq.(1), parameters $\varepsilon_{0}, \phi_{\lambda}$ and $I_{s a t}$ are temperature dependent. While $\phi_{\lambda}$ decreases with temperature due to collisional quenching, the saturation intensity $I_{\text {sat }}$ follows an inverse trend [8]. As a result, fluorescent dyes can lose some of their temperature sensitivity at high laser irradiance. In the following, fluorescein disodium (FL) is used as its temperature sensivity arises exclusively from $\varepsilon_{0}$ and therefore it retains a high and unchanged temperature sensitivity regardless of the laser irradiance [8]. FL is mixed with sulforhodamine 640 (SR640) whose fluorescent emission does not vary with temperature. Figure 3 shows the detection bands selected for the experiments. In the region [ $540 \mathrm{~nm}-560 \mathrm{~nm}$ ], only FL has a contribution to the signal while the emission of SR640 is predominant above $640 \mathrm{~nm}$. Taking the ratio of the signals detected in the two emission bands allows to eliminate the dependence on the droplet shape, since the fluorescence emitted in the two detection bands is affected almost identically by light dispersion at the droplet surface. The ratio $R$ of the signals in the detection bands can be determined by:

$$
R=R_{0} \frac{\varepsilon_{0, \mathrm{FL}}(T)}{\varepsilon_{0, \mathrm{FL}}\left(T_{0}\right)}
$$

where $R_{0}$ is a reference ratio measured at a known temperature $T_{0}$. The fluorescence ratio $R$ calculated by dividing the images of the two cameras is converted into temperature using a calibration curve. The calibration was carried out in a glass cell in which the fluorescent solution was heated progressively from $20^{\circ} \mathrm{C}$ to $80^{\circ} \mathrm{C}$. Images were recorded in the two detection bands and the fluorescence ratio was determined. For a mixture $C_{\mathrm{SR} 640}=0.7 \cdot 10^{-6} \mathrm{M}$ and $C_{\mathrm{FL}}=2 \cdot 10^{-4} \mathrm{M}$ (used later to investigate the drop impact), the temperature sensitivity of the fluorescence ratio $R$ can be valuated at $2.7 \% /{ }^{\circ} \mathrm{C}$, meaning that $R / R_{0} \approx \exp \left\{-0.027\left(T-T_{0}\right)\right\}$. An offset of a few pixels between the images of the two cameras may remain despite a careful alignment of the beamsplitter. A polynomial transform is created to obtain a perfect matching between the coordinates of the pixels in the images of the two cameras. This polynomial function is determined with the help of a dotted grid placed in front of the detection system. In Eq.(2), $T_{0}$ is assimilated to the temperature measured by the thermocouple inserted in the thermal shield (Figure 1). It is assumed that the droplets do not heat up significantly when they travel in the hot air plume above the sapphire window.

\section{Measurements of the temperature and heat flux at the solid surface}

The temperature of the impact surface is characterized by means of an IR camera (FLIR ORION SC7000) which incorporates a cooled InSb detector that operates in the 1.5- to 5.5- $\mu \mathrm{m}$ waveband. The camera is capable of capturing up to $400 \mathrm{fps}$ at full resolution (320 x 240 pixels). Windowing makes acquiring the images at a few thousands fps possible, however at the expense of the number of pixels. Bottom view images of the impact surface can be recorded thanks to the transparency of the sapphire substrate below $5 \mu \mathrm{m}$. The IR camera is equipped with a high-magnification lens with a large numerical aperture. The acquisition sequence is triggered by the passage of the droplet through the optical barrier. The top face of the sapphire window is coated with a thin film of TiAIN (typical thickness: $300 \mathrm{~nm}$ ) which is resistant to the oxidation and stress encountered at high temperature. Furthermore, this coating has a high emissivity in the detection band of the camera $(\epsilon=0.926)$ which is almost independent of temperature. A first benefit of a high emissivity is that the radiative emission from the surface is sufficiently large to shorten the integration time of the camera to a few tens of $\mu s$ and thus to resolve temporally the impact process. Because of the high surface emissivity, reflections of environment radiations (especially those coming from the heated holder) are very limited and can be safely ignored in the processing of the images. The level of the random noise does not exceed $0.1 \%$ of the signal which yields an error of about $0.5^{\circ} \mathrm{C}$ on the evaluation of the temperature. This error remains acceptable in comparison to the temperature variations of the solid surface observed during an impact, which are typically of the order of a few tens of degree. 
Experimentally, a windowing is used to increase the acquisition frame rate of the camera. A windows of $60 \times 80$ pixels was chosen allowing a frame rate of $3.5 \mathrm{kfps}$. In a first test, a non-uniformity correction (NUC) is performed in order to obtain a flat field without any dispersion of the numerical level when there is no droplet and that the conditions are perfectly stationary. A good approximation of the variation in temperature of the wall surface for each pixel $i$ in the image can be expressed by:

$$
\Delta T_{i}(t)=T_{i}\left(t=0^{-}\right)\left[\Delta N_{i}(t) / N_{i}\left(t=0^{-}\right)\right]^{1 / 4}
$$

This expression implies that the camera is linear in flux and $\epsilon$ remains constant with time but knowing the value of $\epsilon$ is not necessary given that the temperature before impact $T_{i}\left(t=0^{-}\right)$is known (the wall temperature being regulated at $600^{\circ} \mathrm{C}$ ). The wall heat flux is deduced from the wall temperature measurement thanks to an inversion of an axisymmetric analytical heat conduction problem. The geometry of the problem is presented in Figure 4. Under the hypothesis of axysymmetry, the transient heat transfer equation can be written:

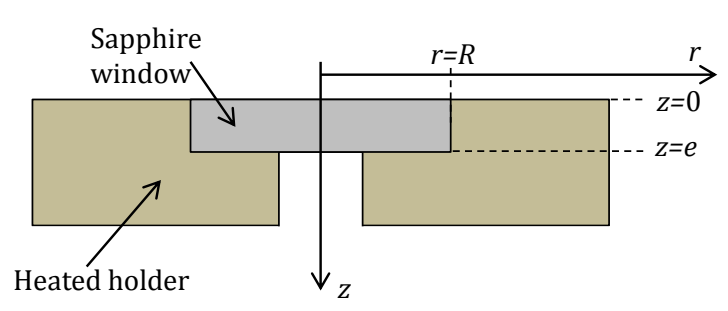

Figure 4. : Geometry of the system

$$
\frac{\partial^{2} T}{\partial r^{2}}+\frac{1}{r} \frac{\partial T}{\partial r}+\frac{\partial^{2} T}{\partial z^{2}}=\frac{1}{a} \frac{\partial T}{\partial t}
$$

With the following boundary conditions :

$$
\begin{array}{r}
T=T_{\text {holder }}, \text { at } r=R, \\
\frac{\partial T}{\partial r}=0, \text { at } r=0, \\
T=T_{\text {holder }}, \text { at } z=e, \\
\lambda \frac{\partial T}{\partial z}=q(r, z=0, t), \text { at } z=0
\end{array}
$$

The heat flux $q(r, z=0, t)$ is assumed to be the contribution of the droplet only and it is therefore the target of the inversion. Indeed, the heat flux associated with natural convection is negligible in comparison with the heat flux induced by the impact of the droplet (typically on the order of a few $\mathrm{MW} / \mathrm{m}^{2}$ ). For the inversion, the reduced variable $\theta=T-T_{\text {holder }}$ is introduced then a Hankel transform in space is applied $(\widetilde{*})$ [9]. This yields:

$$
\widetilde{\theta_{n}}(z=0, t)=\widetilde{Z_{n}}(t) \otimes \widetilde{q_{n}}(z=0, t)
$$

This equation establishes a relationship between the $n^{t h}$ mode of the temperature at the front face $\widetilde{\theta_{n}}(z=0)$ and the $n^{t h}$ mode of the heat flux induced by the drop impact $\widetilde{q_{n}}(z=0)$ in the Hankel transform. $\widetilde{Z_{n}}$ denotes the thermal impedance, which depends on the thermal properties and the geometry of the wall. It can be expressed analytically using in addition to the hankel transform, a Laplace transform in time [9].The number of modes to be considered for the resolution of Eq.(4) is generally chosen using a trial and improvement method. It has to be adapted to the geometry and to the transient period. Presently, 70 modes are considered in the computations. Radial profiles of temperature are extracted from the images assuming a radial symmetry of the problem and the Hankel transformation is applied to them, which allows obtaining $\widetilde{\theta_{n}}(z=0, t)$. Eq.(9) is put in a matrix form for the inversion:

$$
\left[\widetilde{q_{n}}\right]=\left[\widetilde{Z_{n}}\right]^{-1}\left[\widetilde{\theta_{n}}\right] \text { with }\left[\widetilde{\theta_{n}}\right]=\left[\widetilde{\theta_{n}}\left(t_{1}\right), \ldots, \widetilde{\theta_{n}}\left(t_{i}\right), \ldots, \widetilde{\theta_{n}}\left(t_{e n d}\right)\right]
$$

Here, $\left[\widetilde{\theta_{n}}\right]$ is the vector of the temperatures measured at all discrete time steps $t_{1}, \ldots, t_{i}, \ldots, t_{\text {end }}$ of the acquired sequence of images. The last step of the inversion consists in applying the Hankel reverse transformation to get the estimated heat flux in the real space.

\section{Heating of the impacting droplets}

The experiments consisted in varying the falling height of the droplet and thus its impact velocity $V$. The Weber number $W e=\rho V^{2} d / \gamma$, which compares the kinetic energy of the droplet $\left(\sim \rho V^{2}\right)$ to its surface energy $(\sim \gamma / d)$, ranges from 10 to 140 . The wall temperature is set to $600^{\circ} \mathrm{C}$, while the initial droplet temperature is maintained at $20^{\circ} \mathrm{C}$. Figures 5 to 8 show instantaneous images of the temperature taken at different times during the impact. Side and bottom views are recorded for each impact condition. To interpret these images, it should be kept in mind that the imaging system does not provide optical sectioning of the droplet. The images roughly correspond to an average of the temperature in the depth of the droplet given that the depth of field of the cameras is several millimetres. In the following description, the spreading, the recoiling and the fragmentation of the droplet are considered separately.

Spreading: The spreading phase lasts about 3-4 ms. Even if the bulk of the droplet maintains its initial temperature in the early stage of the spreading, there is a thin liquid layer close to the interface with the vapor film, where the liquid temperature quickly reaches about $100^{\circ} \mathrm{C}$. However, this liquid layer is too thin to be observed on the side view images. Its contribution to the bottom views is all the more important than the liquid thickness is small. In the case of $W e=10.2$, the heating is rather moderate during the spreading. In Figure 5, it is difficult to point out a region where the heating is more intense. At higher Weber numbers (Figures 6-8), bottom views show a higher temperature band around the edge of the droplet at $t=1 \mathrm{~ms}$ and $2 \mathrm{~ms}$. At these times, the ejected lamella is 
much thinner than the central region of the droplet, which helps observing a heating of the liquid in the bottom views. Provided a sufficiently large impact velocity, the lamella rapidly takes a gaussian shape surrounded by an annular rim, which is growing due to the deceleration by the surface force opposed to the spreading [10]. Liquid is progressively heated while flowing along the hot wall from the core of the lamella in the direction of the rim at the edge of the droplet. In Figure 8, the liquid temperature apparently reaches almost $80^{\circ} \mathrm{C}$ in the region of minimum thickness just before the entrance of the rim. Afterwards, in the rim, the hot liquid coming from the lamella rapidly mixes with colder liquid already accumulated there. Disturbances on the rim of the spreading drop can be easily observed for $W e=92$ at the early stage of the impact. These disturbances increase with time leading to a spatter with characteristics fingers. Disturbances of smaller amplitudes also develop for lower Weber numbers but much more slowly. Even for $W e=10.2$, the droplet does not keep a perfectly circular shape in the bottom views. It was proposed that fingering is caused by the Rayleigh-Taylor instability because a heavier fluid liquid rim is decelerated with respect to a lighter one air during spreading [11, 12]. The magnitude of the initial deceleration determines the number of fingers and their growth rate. At $t=4 \mathrm{~ms}$ and $5 \mathrm{~ms}$, it can be seen for $W e=92$, both on the side and bottom images, that the fingers are colder than the thinner regions in the rim.

Fragmentation of the rim and splashing: For $W e=92$, the previously mentioned fingers detach from the rest of the rim causing the fragmentation of the rim and ultimately the splashing of the droplet. The temperature of the secondary droplets resulting from the rim fragmentation evolves very little with time. It is of the order of $55^{\circ} \mathrm{C}-60^{\circ} \mathrm{C}$. In parallel, the lamella becomes so thin at the end of the spreading that it breaks. When this occurs, the temperature of the thin liquid sheet (about $80^{\circ} \mathrm{C}$ ) is much larger than that of the surrounding liquid in the rim. Holes usually open in the vicinity of the rim where the lamella is thinner. The holes rapidly expands following the Taylor-Culick theory [13]. During this process, hot liquid ligaments are created because of the opening and expansion of several holes at the same time.

Recoiling and bouncing: The recoiling of the droplet is initiated by the surface forces acting on the rim edge. The case $W e=64.7$ has some similarities with $W e=92$. Here also, several holes open the lamella at the end of the spreading phase. However, the rim does not break up and the empty space replacing the lamella narrows during the recoiling phase. For $W e=10.2$ and $W e=30.6$, the temperature is not uniform when the droplet takes off from the solid surface at about $t=12 \mathrm{~ms}$. For $W e=30.6$, the temperature is lower at the top end where a satellite droplet nearly detaches. In Figure 5, mixing currents are visible at $t=9 \mathrm{~ms}$ and $t=12 \mathrm{~ms}$, while the droplet is about to leave the wall. Hence, the flow induced by the droplet deformation is not capable of a full mixing of the liquid when the impact velocity is weak in the bouncing regime, typically for $W e \leq 30$.

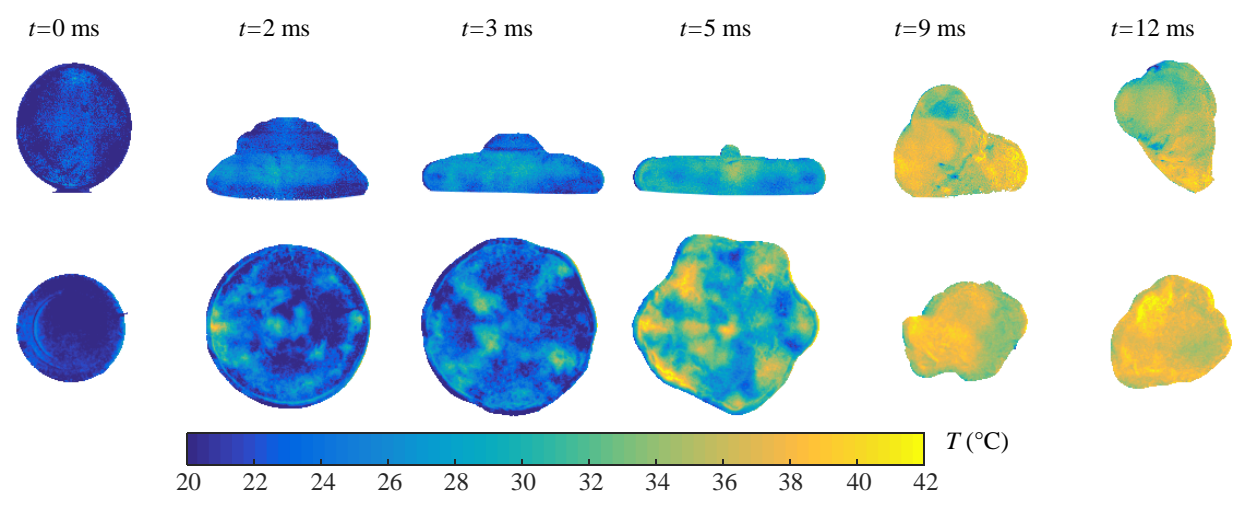

Figure 5. Side and bottom views of the temperature field within an impacting droplet at $W e=10.2$

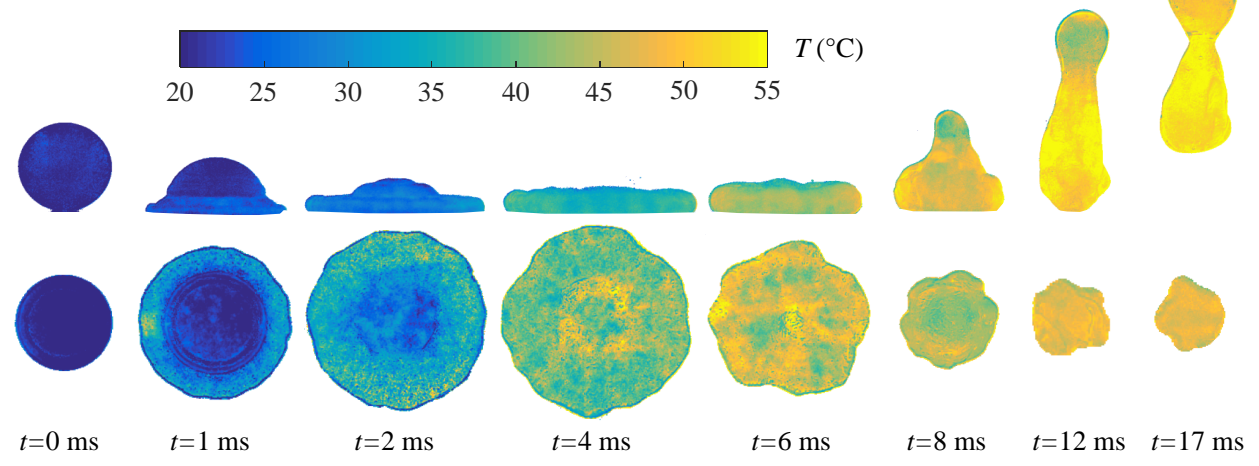

Figure 6. Side and bottom views of the temperature field within an impacting droplet at $W e=30.6$ 


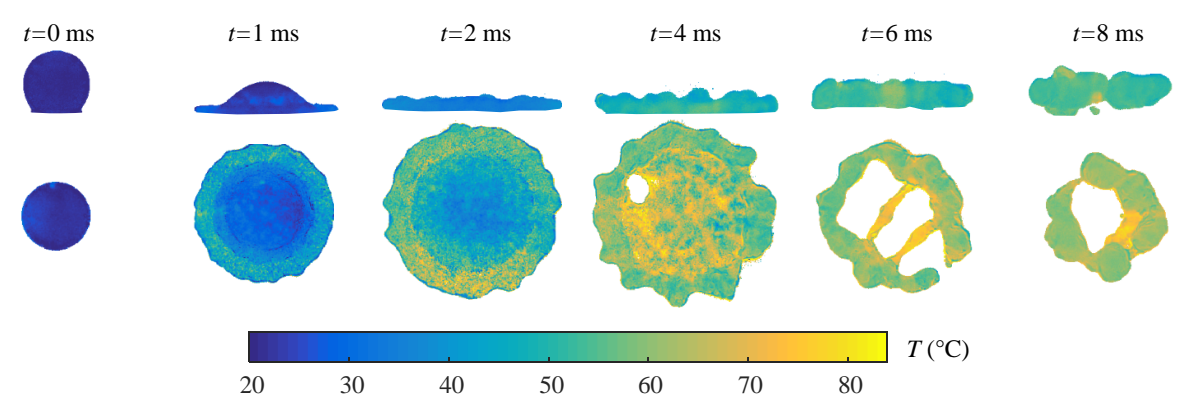

Figure 7. Side and bottom views of the temperature field within an impacting droplet at $W e=64.7$

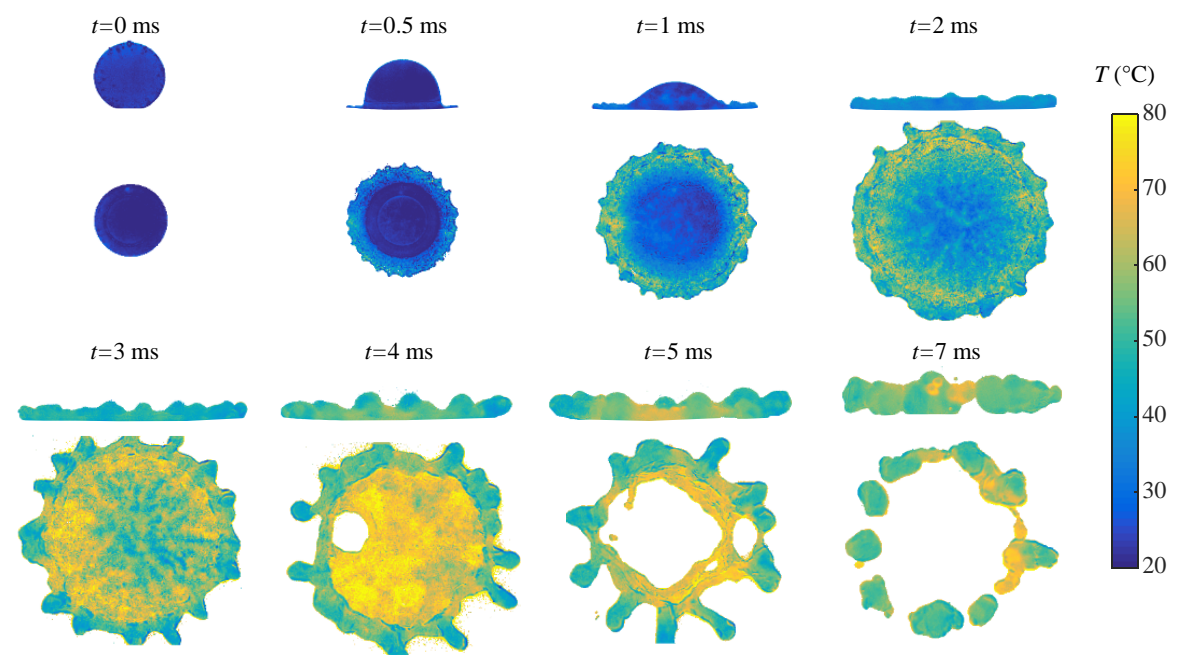

Figure 8. Side and bottom views of the temperature field within an impacting droplet at $W e=92$

\section{Evolution of the heat flux removed at the solid surface}

Figure 9 displays the evolution of the solid surface temperature measured during the impact of a droplet at $W e=$ 140. Measurements situated at the same distance from the droplet center in these images are first averaged. Then, the obtained radial temperature profile is used in the inversion to respect the axial symmetry of the model (Eq.4). The estimated heat flux densities $q(r, z=0)$ is also presented in figure 9 . The heat flux density is maximal shortly before the end of the spreading phase and vanishes progressively thereafter. The obtained values for the heat flux, a few $\mathrm{MW} / \mathrm{m}^{2}$, compare with the literature[3, 4]. The images of the solid surface temperature show some finger-like structures. There are important similarities with the fingering patterns reported by Khavari et al.[14] in the transition boiling regime for the contact area between the droplet and the solid wall. Thus, it may be a reminiscence of the same phenomenon at higher wall temperatures in the film boiling regime.

\section{Influence of the impact velocity}

Figure 10 shows the temporal evolution of the droplet heating for all the tested impact velocities. The reported values are calculated from the spatial averages of the side and bottom images presented in Figures 5-8. Whatever the Weber number, it is observed that the heating evaluated from the bottomview images is systematically larger than that determined from the sideview images. Obviously, both meet at the end of the impingement. Median evolutions, corresponding to the solid curves in Figure 10, are assumed to be closer to the true volumic heating of the droplet. The heating increases with $W e$, but there is a saturation, meaning that $W e$ has almost not influence on the final liquid temperature in the splashing regime contrary to the bouncing regime. This trend has already been observed by Castanet et al.[6] and Dunand et al.[4], who also measured an heating of about $40^{\circ} \mathrm{C}$ in the case of splashing droplets with an initial temperature of $20^{\circ} \mathrm{C}$. The duration of the heating decreases with $W e$. At low impact velocity, the heating period covers both the spreading and the recoiling phases. For $W e=10.2$, the heating is about one third of its final value at the end of the spreading. On the contrary, most of the heating takes place during the spreading for $W e \geq 64.7$. For the high Weber numbers, the heating has reached (or is about to reach) its final value just before that the fragmentation of the rim takes place. Hence, measurements reveal that the fragmentation of the droplet in itself has little effect on the final heating. For comparison, the energy removed from the wall was also calculated by time and space integration of the heat flux determined by the inverse model. The results, shown in Figure 11, are very similar to those presented before for the droplet heating in Figure 10. For instance, the same stagnation of the energy and the same reduction of the period of intense heat exchange can be observed at the high Weber numbers. Both IR and 2cLIF measurements indicate that the heat exchange with the wall is greatly enhanced by the creation of surface under the droplet that takes place during the spreading phase. The creation of surface seems to be the one of the main reason for the differences observed among the experimental cases. In 
addition, the thickness of the vapor film is also influenced by the impact velocity of the droplet as explained in the following.

a)
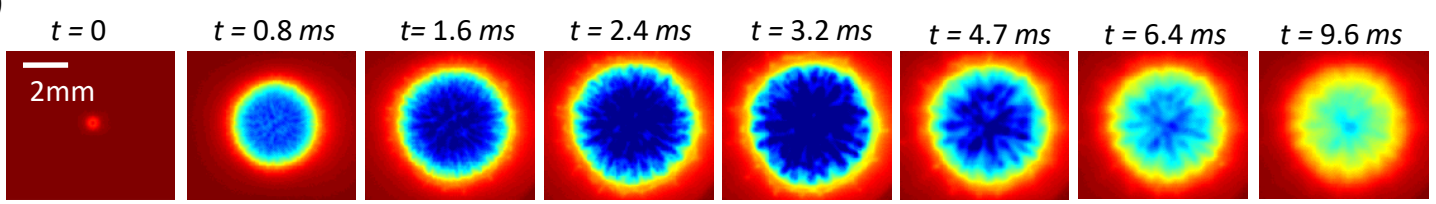

b)
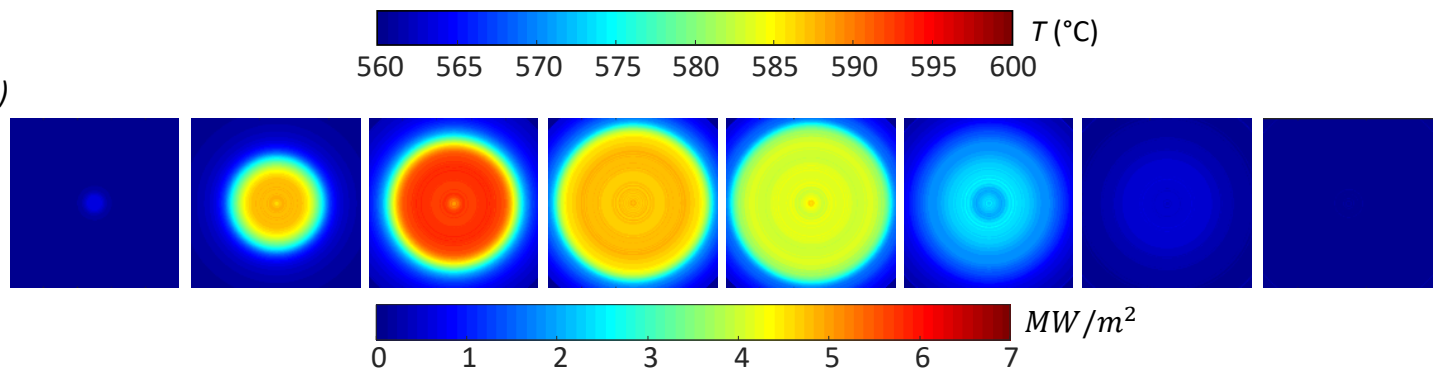

Figure 9. Results of the IR thermography in the case $W e=140$ (a: temperature distribution at the solid surface, $b$ : heat flux density $q(r, z=0)$ derived from the inverse model).

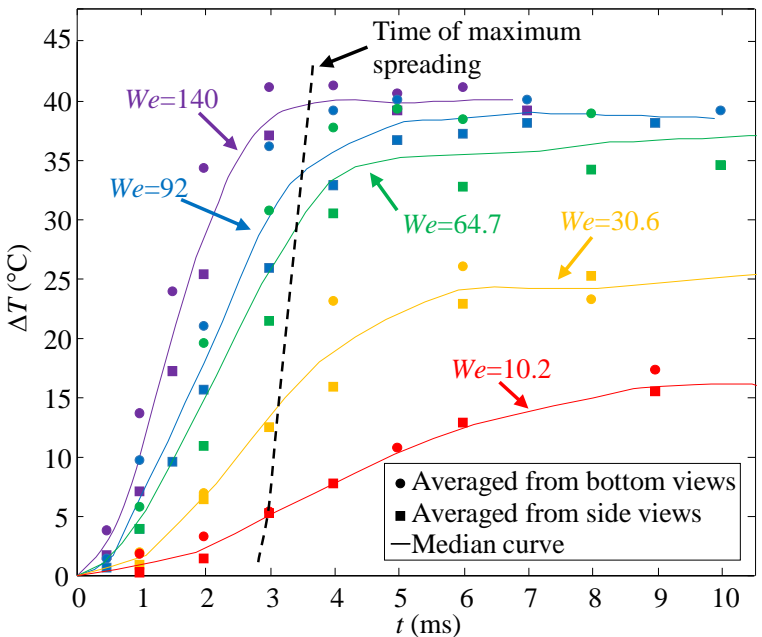

Figure 10. Temporal evolution of the spatially averaged droplet heating

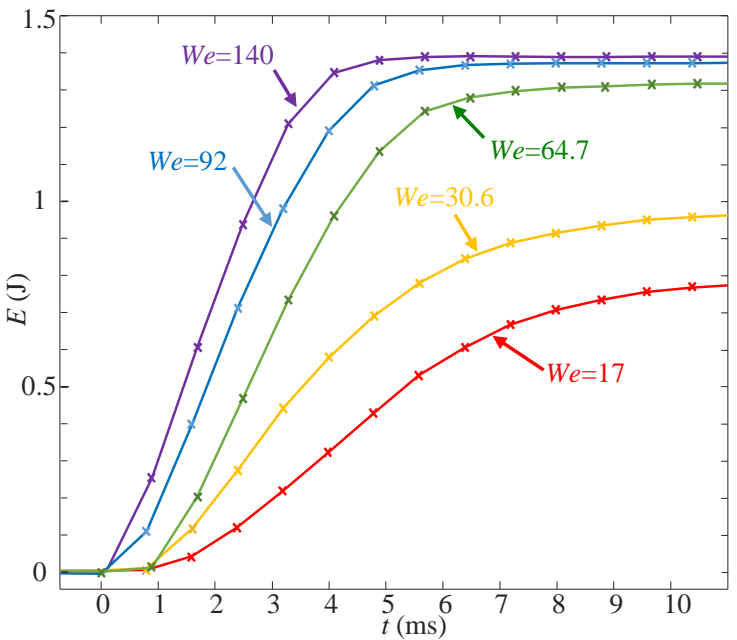

Figure 11. Temporal evolution of the energy removed from the solid surface

The wall heat flux estimated from the IR measurements can be used to evaluate the thickness of the vapor film $h$. Assuming that $h$ is small, even if vapor has a low thermal conductivity, the heat transfer through the vapor film is largely dominated by heat conduction. It can be stated therefore that:

$$
q(r, z=0) \approx \lambda_{v}\left(T_{W}-T_{S A T}\right) / h
$$

where $q(r, z=0)$ is the heat flux extracted at the solid surface, $T_{W}$ the temperature of the solid surface and $T_{S A T}$ the saturation temperature of the liquid equal to $100^{\circ} \mathrm{C}$. The thickness of the vapor film varies spatially and temporally. The thickness of the vapor film $h$ varies in time and space. It takes a minimum value when the heat flux density is maximum. This occurs shortly before the end of the spreading as seen in Figure 9. Figure 12 shows the influence of the Weber number on the minimum thickness of the vapor film $h_{\min }$. As expected, $h_{\min }$ decreases with the impact velocity. The thickness of the vapor film is about 1-2 $\mu \mathrm{m}$, which is typically one order of magnitude lower than the vapor film thickness reported in the literature for sessile droplets (ie. at thermal equilibrium and for $W e=0$ ) [15]. For the high $W e$, the thickness of the vapor film still decreases with $W e$, but very moderately.

Finally, the emphasis was placed onto the comparison between the contributions to the energy heat balance. The heat removed from the wall $E_{\text {solid }}$ and the sensible heat transferred to the liquid $E_{\text {liquid }}$ are determined at the end of the impacts based on the measurements. The difference between these two quantities is related to the energy $E_{\text {vapor }}$ used to evaporate a mass flow $\dot{m}$ of liquid during the impact:

$$
E_{\text {vapor }}=L_{v} \int \dot{m} d t=\frac{1}{1+J a}\left(E_{\text {solid }}-E_{\text {liquid }}\right)
$$

where $J a$ is the Jakob number defined by $J a=H\left(T_{f i l m}\right) / L\left(T_{S A T}\right), H(T)=\rho_{v} C p_{v} T$ et $L(T)=\rho_{v}\left(L_{v}+C p_{v} T\right)$ and $T_{f i l m}=\left(T_{W}+T_{S A T}\right) / 2$. Here, $J a$ is about equal to 0.35 . In Figure $13, E_{\text {solid }}, E_{\text {liquid }}$ et $E_{\text {vapor }}$ are displayed 
as a function of the Weber number. The value of $E_{\text {solid }}$ and $E_{\text {liquid }}$ are very close whatever the Weber number. Hence, almost all of the energy removed from the wall is used to heat up the liquid. Evaporation typically accounts for less than $10 \%$ of the heat taken from the solid wall. The variations observed for $E_{\text {vapor }}$ in Figure 13 are not really significant because $E_{\text {vapor }}$ compares to the experimental uncertainty estimated at about $0.1 \mathrm{~J}$.

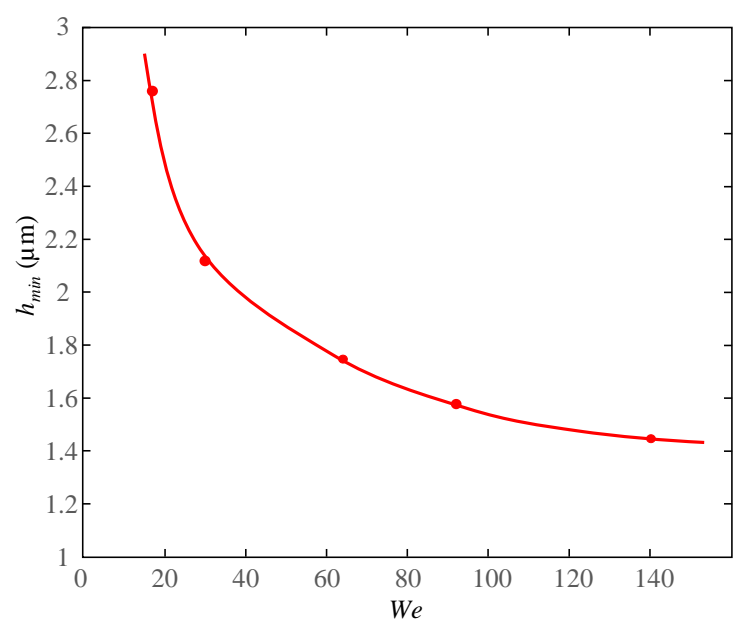

Figure 12. Influence of the Weber number on the minimum thickness of the vapor film

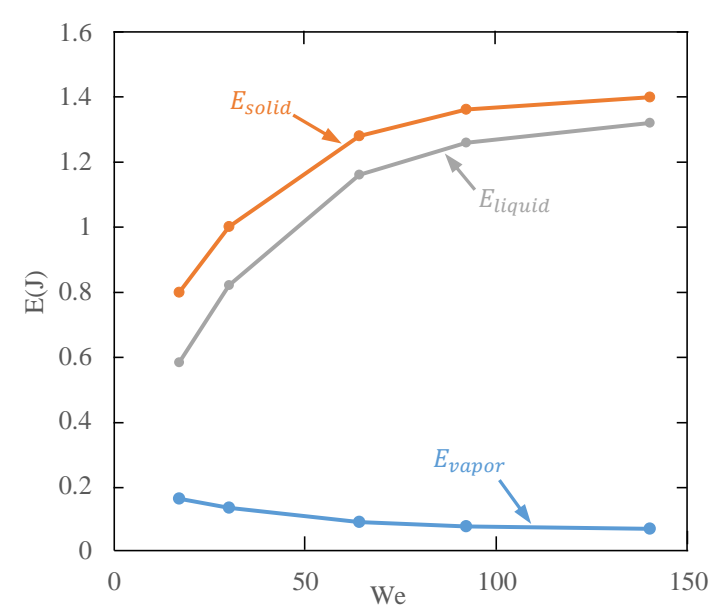

Figure 13. Influence of the Weber number on the heat exchanged between the solid surface, the liquid droplet and the vapor layer

\section{Conclusions}

The heating of the impacting droplet and the cooling of the solid surface in the film boiling regime were quantified accurately using two non-intrusive optical techniques. Measurements show an important effect of the Weber number on the heat transfers. As the Weber number increases, the exchange surface increases and the thickness of the vapor film decreases, which induces an increase in the heat removed from the wall and in the heating of the liquid. However, when increasing the Weber number, the influence of the impact velocity becomes less and less noticeable. There is almost a saturation of the heat extracted from the solid surface in the splashing regime of impact. In the case of the millimeter-sized droplets considered in this study, almost all the cooling of the solid wall results from the sensible heating of the liquid. This shows the great interest in modelling the deformation and the thermal convection within the impacting droplets to predict the intensity of the wall cooling.

\section{Acknowledgements}

The authors acknowledge the financial support of the Lorraine region through the CPER ENERBATIN projet.

\section{References}

[1] Liang, G., and Mudawar, I., 2017. International Journal of Heat and Mass Transfer, 106, pp. 103 - 126.

[2] Bernardin, J. D., and Mudawar, I., 1997. International Journal of Heat and Mass Transfer, 40(11), pp. 2579 2593.

[3] Jung, J., Jeong, S., and Kim, H., 2016. International Journal of Heat and Mass Transfer, 92, pp. $774-783$.

[4] Dunand, P., Castanet, G., Gradeck, M., Maillet, D., and Lemoine, F., 2013. International Journal of Heat and Fluid Flow, 44, pp. 170-180.

[5] Lemoine, F., and Castanet, G., 2013. Experiments in Fluids, 54(7).

[6] Castanet, G., Lienart, T., and Lemoine, F., 2009. International Journal of Heat and Mass Transfer, 52(3-4), pp. 670-679.

[7] Dunand, P., Castanet, G., and Lemoine, F., 2012. Experiments in Fluids, 52(4), pp. 843-856.

[8] Chaze, W., Caballina, O., Castanet, G., and Lemoine, F., 2016. Experiments in Fluids, 57(4), p. 58.

[9] Gradeck, M., Ouattara, J., Rémy, B., and Maillet, D., 2012. Experimental Thermal and Fluid Science, 36, pp. $56-64$.

[10] Castanet, G., Caballina, O., and Lemoine, F., 2015. Physics of Fluids, 27(6), p. 063302.

[11] Thoroddsen, S. T., and Sakakibara, J., 1998. Physics of Fluids, 10(6), pp. 1359-1374.

[12] Mehdizadeh, N. Z., Chandra, S., and Mostaghimi, J., 2004. Journal of Fluid Mechanics, 510, pp. $353-373$.

[13] Culick, F. E. C., 1960. Journal of Applied Physics, 31(6), pp. 1128-1129.

[14] Khavari, M., Sun, C., Lohse, D., and Tran, T., 2015. Soft Matter, 11, pp. 3298-3303.

[15] Celestini, F., Frisch, T., Cohen, A., Raufaste, C., Duchemin, L., and Pomeau, Y., 2014. Physics of Fluids, 26(3), p. 032103. 